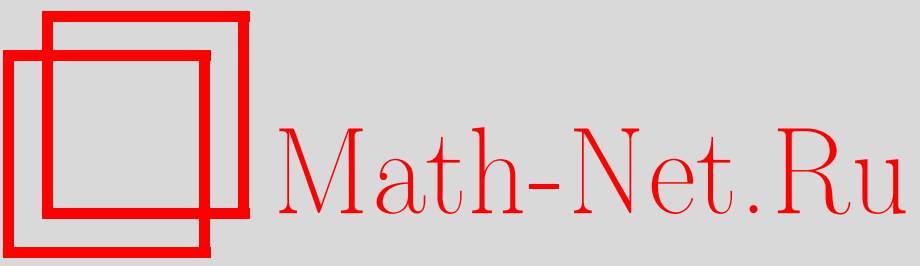

И. П. Шепеть, Д. Б. Литвин, Синтез дуального программного управления многомерной динамической системой, Итоги науки и техн. Сер. Соврем. мат. и ее прил. Темат. обз., 2019, том 167, 117-123

DOI: https://doi.org/10.36535/0233-6723-2019-167-117-123

Использование Общероссийского математического портала Math-Net.Ru подразумевает, что вы прочитали и согласны с пользовательским соглашением

http: //www.mathnet.ru/rus/agreement

Параметры загрузки:

IP: 52.90 .164 .192

26 апреля 2023 г., 13:07:09 


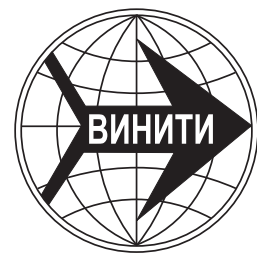

ИТОГИ НАУКИ И ТЕХНИКИ.

Современная математика и ее приложения.

Тематические обзоры.

Том 167 (2019). С. 117-123

DOI: 10.36535/0233-6723-2019-167-117-123

УДК 517.977

\title{
СИНТЕЗ ДУАЛЬНОГО ПРОГРАММНОГО УПРАВЛЕНИЯ МНОГОМЕРНОЙ ДИНАМИЧЕСКОЙ СИСТЕМОЙ
}

\author{
(c) 2019 г. $\quad$ И. П. ШЕПЕТЬ, Д. Б. ЛИТВИН
}

\begin{abstract}
АннотАция. Рассмотрена постановка задачи синтеза дуального программного управления многомерной динамической системой. Определены различные варианты показателей качества дуального программного управления. На основе метода Релея-Ритца синтезирован закон управления пространственным положением блока чувствительных элементов инерциальной навигационной системы.
\end{abstract}

Ключевъе слова: многомерная динамическая система, дуальное программное управление, закон управления, показатель качества, система стохастических дифференциальных уравнений.

\section{SYNTHESIS OF DUAL PROGRAM CONTROL FOR A MULTIDIMENSIONAL DYNAMICAL SYSTEM}

\author{
(c) 2019 I. P. SHEPET, D. B. LITVIN
}

\begin{abstract}
In this paper, we consider the problem of synthesis of dual program control for a multidimensional dynamic system and propose various quality indicators for dual program control. Based on the Rayleigh-Ritz method, we synthesize a control law for the spatial position of the block of sensitive elements of an inertial navigation system.
\end{abstract}

Keywords and phrases: multidimensional dynamical system, dual program control, control law, quality indicator, system of stochastic differential equations.

AMS Subject Classification: 49M27, 65K10

Существуют задачи синтеза стохастического управления по неполной информации, минимизирующего среднее значение функции потерь в условиях, когда точность оценки текущих фазовых координат зависит от вектора управления (см. [5]). В этих условиях стохастическое управление дуально, так как выбирает разумный компромисс между стремлениями в каждой реализации уменьшить функцию потерь и увеличить точность оценки. Примерами задач синтеза оптимального дуального управления могут служить следующие задачи: согласование (физическое и аналитическое) измерительного и опорного трехгранников инерциальных навигационных систем (ИНС) (начальная выставка); оценка параметров ИНС и идентификация погрешностей чувствительных элементов, зависящих от компонентов вектора управления - пространственного положения чувствительных элементов, при коррекции от датчиков внешней информации (позиционных, скоростных, угловых); идентификация погрешностей чувствительных элементов при наземной калибровке.

Рассмотрим общую постановку задачи синтеза дуального управления динамической системой, возмущаемой случайными шумами, в условиях, когда измерения фазовых координат системы или зависящих от них величин производится со случайными ошибками (см. $[1,5])$. 
Управление производится на отрезке времени $\left[t_{0}, T\right]$, где $t_{0}, T$ заданы. $n$-Мерный вектор фазовых координат $x$ динамической системы удовлетворяет уравнению

$$
\dot{x}=f(x, u, w, t),
$$

где $u-m$-мерный вектор управления, $w-l$-мерный вектор случайных шумов, возмущающих систему, $f$-заданная вектор-функция соответствующего числа переменных. В моменты времени $t_{0}, \ldots, t_{k}, \ldots, t_{N}\left(t_{N} \leqslant T\right)$ измеряются векторы $y_{0}, \ldots, y_{k}, \ldots, y_{N}$, несущие информацию о векторе $x$. Векторы $y_{k}$ имеют вид

$$
y_{k}=\left(t_{k}, x_{k}, \xi_{k}\right)
$$

где $x_{k}=x\left(t_{k}\right), \xi_{k}-r$-мерный дискретный случайный процесс ошибок измерений, $H$ - заданная вектор-функция соответствующего числа переменных.

Общим показателем качества управления многомерным стохастическим объектом является средний риск (см. [1]):

$$
R=M\left[\int_{0}^{T} L(\tau, \delta X(\tau), u(\tau)) d \tau\right]
$$

где $M$ - символ математического ожидания; $u(t)$ - вектор управления; $\delta X(\tau)$ - вектор ошибок управления; $L-$ функция потерь, характеризующая потери в каждый момент времени $\tau$ за счет отклонения вектора выходного сигнала системы от требуемого значения, а также за счет расходования ресурсов на управление.

При синтезе оптимального управления должен быть определен вектор $u_{k}(\tau)$, который в каждой конкретной реализации процесса управления должен быть неслучайной функцией $\tau$ и векторов измерений $y_{0}, \ldots, y_{k}, \ldots$ (см. $\left.[1,5]\right)$. Однако можно поставить задачу управлять системой так, чтобы получить вектор оценки, оптимальный на множестве допустимых управлений, т.е. оптимальные управления выбирать из условия достижения наилучшей оценки фазовых координат системы.

Рекуррентные уравнения оптимизации, векторы оптимальных оценок и управлений в виде пригодном для решения прикладных задач, удается получить при выполнении следующих условий:

1. Условная плотность вероятности $p\left(x_{k} \mid y_{k}, \ldots, y_{0}\right)$ - носитель всей информации о векторе $x_{k}$, содержащейся в векторах измерений $y_{k}, \ldots, y_{0}$, может быть представлена некоторой известной функцией от $x_{k}$ и вектора $B_{k}$, зависящего от $y_{k}, \ldots, y_{0}$ :

$$
p\left(x_{k} \mid y_{k}, \ldots, y_{0}\right)=p_{k}\left(x_{k} \mid B_{k}\right)
$$

где $B_{k}=B_{k}\left(y_{k}, \ldots, y_{0}\right)$. Вектор $B_{k}$ совпадает с определяемым в математической статистике определением исчерпывающей системой статистик (вектором достаточных статистик). На множестве векторов $y_{k}, \ldots, y_{0}$, сохраняющем постоянным вектор $B_{k}$, распределение вектора $x_{k}$ остается постоянным.

2. При фиксированных векторах $u_{k}$ последовательность векторов $B_{k}$ является марковской:

$$
p\left(B_{k+1} \mid B_{k}, \ldots, B_{0}\right)=p_{k}\left(B_{k+1} \mid B_{k}\right) .
$$

При решении прикладной задачи поиска пространственного положения блока чувствительных элементов (БЧЭ) управляемой инерциальной навигационной системы (УИНС) оба этих условия выполняются (см. [7]). 
Вектор оптимальной оценки фазовых координат $\hat{x}\left(B_{N}\right)$ и векторы оптимальных управлений $u_{N-1}^{0}\left(B_{N-1}\right), \ldots, u_{0}^{0}\left(B_{0}\right)$ определяется при последовательном решении уравнений

$$
\begin{aligned}
& R_{N}^{0}\left(B_{N}\right)=\inf \left\{\int L(x, \hat{x}) p_{N}\left(x \mid B_{N}\right) d x \mid \hat{x} \in X\right\}, \\
& R_{N-1}^{0}\left(B_{N-1}\right)=\inf \left\{\int R_{N}^{0}(B) p_{N}\left(B \mid B_{N-1}, u\right) d B \mid u \in U_{N-1}\right\}, \\
& R_{0}^{0}\left(B_{N-1}\right)=\inf \left\{\int R_{1}^{0}(B) p_{1}\left(B \mid B_{0}, u\right) d B \mid u \in U_{0}\right\} .
\end{aligned}
$$

Векторы оптимальных управлений $u_{N-1}^{0}\left(B_{N-1}\right), \ldots, u_{0}^{0}\left(B_{0}\right)$ назовем векторами дуалъных управлений (см. $[1,5])$.

При попытке решения рекуррентных уравнений оптимизации (4)-(6) возникают непреодолимые трудности, связанные с нахождением условных плотностей вероятностей и выражений для минимальных условных рисков. Преодоление указанных трудностей возможно при представлении управляющих воздействий или траекторий вектора состояния в виде такой последовательности функций, которые обеспечивают последовательно все меньшие значения минимизируемого функционала. Данный метод может найти широкое применение в ИНС с управляемым блоком чувствительных элементов.

Задача инерциальной курсовертикали как элемента ИНС - формировать опорную систему координат. Решение этой задачи осуществляется реализацией невозмущаемого маятника Шулера на основе интегральной коррекции. Появление акселерометров и гироскопов, работающих на новых физических принципах, позволили перейти к аналитическому формированию опорной системы координат и тем самым расширили возможности автономной компенсации погрешностей чувствительных элементов путем управления пространственным положением БЧЭ.

Погрешности чувствительных элементов ИНС (гироскопов и акселерометров) приводит к отклонению измерительной системы координат от опорной и, как следствие, возникновению ошибок определения навигационных параметров подвижного объекта.

Вектор ошибок определения навигационных параметров описывает управляемый процесс и описывается системой линейных стохастических дифференциальных уравнений:

$$
\delta \dot{x}^{*}=F^{*} \delta x^{*}+G w,
$$

где $\delta x^{*}=\left[\delta x_{u}^{*}, \delta x_{b}\right]^{T}$ - расширенный вектор состояния ошибок управляемой ИНС; $\delta x_{u}^{*}$-вектор ошибок выходных параметров управляемой ИНС; $\delta x_{\text {в }}$ - вектор возмущающих воздействий.

Элементы матрицы $F^{*}$ зависят от параметров углового движения БЧЭ относительно опорной системы координат $(\alpha, \dot{\alpha}, \beta, \dot{\beta}, \chi, \dot{\chi})$. Следовательно, и выходные ошибки системы (т.е. элементы вектора $\left.\delta x_{u}^{*}\right)$ зависят от программы вращения БЧЭ.

Для систем, подверженных случайным воздействиям, применяются статистические показатели оптимизации, с помощью которых оценивают выходные эффекты в среднем для большого числа реализаций процессов на входах системы (см. $[1,5])$. Качество управления для таких систем характеризуется величиной среднего риска (3), представляющего собой безусловное математическое ожидание функции потерь. С учетом соотношения (7) имеем:

$$
R=M\left[\int_{t_{0}}^{t_{R}} L\left(\tau, \delta x_{u}^{*}, u(\tau)\right) d \tau\right]=\int_{t_{0}}^{t_{R}}\left(\left[L\left(\tau, \delta x_{u}^{*}, u(\tau)\right)\right]\right) d \tau .
$$

Погрешности вектора навигационных параметров, вырабатываемые управляемой ИНС, имеют различную размерность. Для того чтобы оперировать соизмеримыми величинами, введем в рассмотрение нормированный вектор ошибок навигационных параметров:

$$
x_{u}^{\mathrm{H}}=\delta x_{u}^{*}(\tau),
$$


где $C$ - нормирующая матрица.

Ковариационная матрица вектора $\delta x_{u}^{\mathrm{H}}$ определяется в соответствии с выражением

$$
P_{\mathrm{H}}=C P_{u} C^{T}
$$

(см. [4]), где $P_{u}=M\left[\delta x_{u}^{* T} \delta x_{u}^{*}\right]$ - ковариационная матрица ошибок управляемой ИНС.

Преобразуем подынтегральное выражение в (8) с учетом соотношений (9) и (??). Имеем

$$
M\left[L_{A}\left(\tau, \delta x_{u}^{*}\right)\right]=L_{A}^{\mathrm{H}}\left[\tau, P_{\mathrm{H}}\right] .
$$

В зависимости от вида функции $L_{A}^{\mathrm{H}}\left[\tau, P_{\mathrm{H}}\right]$, имеют место следующие показатели качества. При

$$
L_{A}^{\mathrm{H}}\left[\tau, P_{\mathrm{H}}\right]=\lambda_{\max }\left\{P_{\mathrm{H}}\right\},
$$

где $\lambda_{\max }\left\{P_{\mathrm{H}}\right\}$ - максимальное характеристическое число матрицы $P_{\mathrm{H}}$, подынтегральное выражение в показателе качества (8) задает максимальную ось корреляционного эллипсоида рассеяния. При минимизации показателя (8) минимизируется значение дисперсии ошибки одного параметра в векторе нормированных параметров, а именно, имеющего максимальную величину дисперсии. Если

$$
L_{A}^{\mathrm{H}}\left[\tau, P_{\mathrm{H}}\right]=\operatorname{tr}\left\{P_{\mathrm{H}}\right\},
$$

где $\operatorname{tr}\left\{P_{\mathrm{H}}\right\}$ - след матрицы $P_{\mathrm{H}}$, то подынтегральное выражение в показателе качества (8) задает среднюю дисперсию вектора $z$ (величину диагонали ориентированного вдоль центральных осей системы координат параллелепипеда, в который вписывается эллипсоид рассеяния).

Показатель качества (8) с подынтегральным выражением (12) эквивалентен классическому интегрально-квадратическому функционалу с диагональной матрицей коэффициентов (см. [3]):

$$
R_{A}=M\left[\int_{t_{0}}^{t_{R}}\left(\delta x_{u}^{* T}(\tau) Q_{x} \delta x_{u}^{*}(\tau)\right) d \tau\right]
$$

где $Q_{x}$ - диагональная матрица коэффициентов потерь. В этом случае интеграл (13) будет учитывать суммарные погрешности управляемой ИНС за время $t_{R}-t_{0}$.

Выражение (13) можно представить в виде:

$$
\begin{aligned}
R_{A}=M\left[\int_{t_{0}}^{t_{R}}\left(\sum_{i=1}^{n} q_{x i i} \delta x_{u i}^{*}(\tau) \delta x_{u i}^{*}(\tau)\right) d \tau\right]= \\
\quad=\int_{t_{0}}^{t_{R}}\left(M\left[\sum_{i=1}^{n} q_{x i i} \delta x_{u i}^{*}(\tau) \delta x_{u i}^{*}(\tau)\right]\right) d \tau=\int_{t_{0}}^{t_{R}}\left(\sum_{i=1}^{n} q_{x i i} M\left[\delta x_{u i}^{*}(\tau) \delta x_{u i}^{*}(\tau)\right]\right) d \tau
\end{aligned}
$$

где $n$ - размерность вектора навигационных параметров управляемой ИНС.

Выражение $M\left[\delta x_{u i}^{*}(\tau) \delta x_{u i}^{*}(\tau)\right]$ в $(14)$ представляет собой диагональный элемент ковариационной матрицы $P_{u i j}(\tau)$ ошибок навигационных параметров управляемой ИНС. Получаем

$$
R_{A}=\int_{t_{0}}^{t_{R}}\left(\sum_{i=1}^{n} q_{x i i} P_{u i i}(\tau)\right) d \tau=\int_{t_{0}}^{t_{R}}\left(\operatorname{tr}\left\{Q_{x} P\right\}\right) d \tau=\int_{t_{0}}^{t_{R}}\left(\operatorname{tr}\left\{P_{\mathrm{H}}\right\}\right) d \tau,
$$

что подтверждает эквивалентность показателя (15) и (8) с подынтегральным выражением (12).

Рассмотрим случай, когда

$$
L_{A}^{\mathrm{H}}\left[\tau, P_{\mathrm{H}}\right]=\operatorname{det}\left\{P_{\mathrm{H}}\right\},
$$

где $\operatorname{det}\left\{P_{\mathrm{H}}\right\}$ - определитель матрицы $P_{\mathrm{H}}$. При этом подынтегральное выражение в показателе качества (8) задает обобщенную дисперсию вектора ошибок ИНС (объем эллипсоида рассеяния, пропорциональный квадрату этой дисперсии). Соотношение (16) определяет количество шенноновской информации о векторе ошибок ИНС (см. [2]). 
Остановимся на выборе нормирующей матрицы $C$. Значения элементов нормирующей матрицы должны иметь четкий физический смысл. В качестве элементов нормирующей матрицы необходимо выбрать коэффициенты, зависящие от погрешностей некоторой базовой (эталонной) системы. В качестве такой базы может служить неуправляемая ИНС, работающая в тех же условиях, что и управляемая ИНС. Поставим каждому элементу вектора $\delta x_{u}^{*}(\tau)$ в соответствие определенный элемент вектора $\delta x_{u}^{\mathrm{H}}(\tau)$ на основании правила

$$
\delta x_{u i}^{\mathrm{H}}(\tau)=\frac{1}{\sqrt{P_{i i}^{0}(\tau)}} \delta x_{u i}^{*}(\tau),
$$

где $\delta x_{u i}^{\mathrm{H}}(\tau)-i$-й элемент нормированного вектора ошибок навигационных параметров; $\delta x_{u i}^{*}(\tau)-$ $i$-й элемент вектора ошибок навигационных параметров; $P_{i i}^{0}(\tau)$ - диагональные элементы ковариационной матрицы ошибок неуправляемой ИНС. Тогда нормирующая матрица будет иметь вид

$$
C=\left[\begin{array}{cccc}
\frac{1}{\sqrt{P_{11}^{0}(\tau)}} & 0 & \ldots & 0 \\
0 & \frac{1}{\sqrt{P_{22}^{0}(\tau)}} & \cdots & 0 \\
\vdots & \vdots & \ddots & \vdots \\
0 & 0 & \ldots & \frac{1}{\sqrt{P_{10,10}^{0}(\tau)}}
\end{array}\right]
$$

Ковариационная матрица нормированного вектора $\delta x_{u}^{i}$ ошибок навигационных параметров имеет вид

$$
P_{r}=\left[\begin{array}{ccccc}
\frac{P_{u 11}(\tau)}{P_{11}^{0}(\tau)} & \frac{P_{u 12}(\tau)}{\sqrt{P_{11}^{0}(\tau) P_{22}^{0}(\tau)}} & \frac{P_{u 13}(\tau)}{\sqrt{P_{11}^{0}(\tau) P_{33}^{0}(\tau)}} & \cdots & \frac{P_{u 110}(\tau)}{\sqrt{P_{11}^{0}(\tau) P_{1010}^{0}(\tau)}} \\
\frac{P_{u 21}(\tau)}{\sqrt{P_{22}^{0}(\tau) P_{11}^{0}(\tau)}} & \frac{P_{u 22}(\tau)}{P_{22}^{0}(\tau)} & \frac{P_{u 23}(\tau)}{\sqrt{P_{22}^{0}(\tau) P_{33}^{0}(\tau)}} & \cdots & \frac{P_{u 210}(\tau)}{\sqrt{P_{22}^{0}(\tau) P_{1010}^{0}(\tau)}} \\
\frac{P_{u 31}(\tau)}{\sqrt{P_{33}^{0}(\tau) P_{11}^{0}(\tau)}} & \frac{P_{u 32}(\tau)}{\sqrt{P_{33}^{0}(\tau) P_{22}^{0}(\tau)}} & \frac{P_{u 33}(\tau)}{P_{33}^{0}(\tau)} & \cdots & \frac{P_{u 310}(\tau)}{\sqrt{P_{33}^{0}(\tau) P_{1010}^{0}(\tau)}} \\
\vdots & \vdots & \vdots & \ddots & \vdots \\
\frac{P_{u 101}(\tau)}{\sqrt{P_{1010}^{0}(\tau) P_{11}^{0}(\tau)}} & \frac{P_{u 102}(\tau)}{\sqrt{P_{1010}^{0}(\tau) P_{22}^{0}(\tau)}} & \frac{P_{u 103}(\tau)}{\sqrt{P_{1010}^{0}(\tau) P_{33}^{0}(\tau)}} & \cdots & \frac{P_{u 1010}}{P_{1010}^{0}}
\end{array}\right] .
$$

Элементы ковариационных матриц $P_{u i j}(t)$ и $P_{i i}^{0}(t)$ могут быть найдены решением следующего дифференциального уравнения:

$$
\begin{gathered}
\dot{P}_{u}(\dot{\chi}, \dot{\alpha}, \dot{\beta}, t)=F^{*}(\dot{\chi}, \dot{\alpha}, \dot{\beta}, t) P_{u}(\dot{\chi}, \dot{\alpha}, \dot{\beta}, t)+P_{u}(\dot{\chi}, \dot{\alpha}, \dot{\beta}, t) F^{* T}(\dot{\chi}, \dot{\alpha}, \dot{\beta}, t)+G H G^{T}, \\
\dot{P}^{0}(t)=F^{0}(t) P^{0}(t)+P^{0}(t) F^{0 T}(t)+G^{0} H^{0} G^{0 T},
\end{gathered}
$$

где $F^{*}$ и $F^{0}$ - матрицы динамики ошибок управляемой и неуправляемой ИНС соответственно; $G$ и $G^{0}$ - матрицы возмущений управляемой и неуправляемой ИНС соответственно; $H$ и $H^{0}-$ матрицы интенсивностей возмущающих воздействий управляемой и неуправляемой ИНС соответственно.

В качестве управления рассмотрим вектор угловых скоростей вращения БЧЭ в пространстве:

$$
u(t)=[\dot{\chi}, \dot{\alpha}, \dot{\beta}]^{T} .
$$


Совокупность уравнений (20) представляет собой объект управления. При этом первое уравнение является системой линейных детерминированных дифференциальных уравнений и представляет собой эволюцию точностных характеристик управляемой ИНС. Ошибки управляемой ИНС определяются уравнением (7) - системой линейных стохастических дифференциальных уравнений. Таким образом, задача поиска оптимального управления для стохастического объекта сведена к задаче управления детерминированным объектом, что позволяет ее квалифицировать как задачу поиска программного управления.

Поиск оптимального, в смысле выбранного функционала качества (8), управления пространственным положением БЧЭ для объекта (20) является задачей нелинейного оптимального управления. В результате ее решения необходимо получить закон пространственного движения БЧЭ относительно одной из контролируемых систем координат, который будет являться функцией времени, так зависящий от априорных представлений о параметрах случайных возмущений, чтобы был наибольший некоторый средний эффект управления (см. [1]). Для многомерных нелинейных по управляемым параметрам систем, каковыми и являются исследуемые ИНС, решение этой задачи встречает огромные трудности. Эти трудности являются принципиальными и связаны с размерностью множеств, с которыми приходится сталкиваться при численном решении уравнений, описывающих объект управления (см. [3]).

Преодоление указанных трудностей возможно на основе использования метода Релея-Ритца, предусматривающего разложение траектории вектора управления в ряд с взвешиваемыми слагаемыми по подходящему набору базисных функций и определение значение коэффициентов этого ряда, минимизирующих исходный функционал.

Проведенные исследования (см. [7]) показывают, что траектория вектора управления близка к периодической и может быть представлены в виде разложения в ряд Фурье. На основании данных исследования был произведен поиск оптимального управления положением БЧЭ в азимуте.

Получены следующие параметры закона управления

$$
\dot{\chi}(t)=\sum_{k=1}^{n} a_{k} \sin (k \omega t)
$$

пространственным положением БЧЭ (см. [6]):

$$
\omega=0,012 \mathrm{c}^{-1}, \quad \frac{a_{1}}{\omega}=132,9^{\circ}, \quad a_{2}=0, \quad \frac{a_{3}}{3 \omega}=2^{\circ}, \quad a_{4}=0, \quad \frac{a_{5}}{5 \omega}=-0,5^{\circ}, \quad a_{6}=0 .
$$

Ошибки управляемой ИНС инвариантны к начальному азимутальному положению подвижного объекта.

Применение управления пространственным положением БЧЭ эффективно для уменьшения ошибок по координатам, скоростям и вертикали. Так, среднеквадратическое отклонение (CKO) ошибки определения координат к концу первого часа работы уменьшается в 3,5-4 раза и не превышает 700 м. СКО ошибки определения скорости не превышает 0,5 м/с за время функционирования ИНС. СКО ошибки определения вертикали к концу первого часа работы системы в 2,5-3,5 раза меньше СКО аналогичной ошибки ИНС без использования эффекта автокомпенсации погрешностей чувствительных элементов.

\section{СПИСОК ЛИТЕРАТУРЫ}

1. Богуславский И. А. Прикладные задачи фильтрации и управления. - М.: Наука, 1983.

2. Ермакова С. М. (ред.). Математическая теория планирования эксперимента. - М.: Наука, 1983.

3. Красовский H. Н. (ред.). Справочник по теории автоматического управления. - М.: Наука, 1987.

4. Малышев B. В., Красильщиков М. Н., Карлов В. И. Оптимизация наблюдения и управления летательных аппаратов. - М.: Машиностроение, 1989.

5. Фельдбаум А. А. Основы теории оптимальных автоматических систем. - М.: ГИФМЛ, 1963.

6. Шепеть И. П. Способ инерциальной навигации и устройство для его осуществления/ Патент 2572403 РФ, МПК G06C 21/12. Заявитель и патентообладатель Шепеть И. П. № 2015111505/28. Заявл. 30.03.2015, опубл. 10.01.2016. 
7. Шепетъ И. П., Онуфриенко В. В., Слесаренок С. В. Методическое обеспечение управляемых навигационных систем. - Воронеж: Военный учебно-научный центр ВВС «Военно-воздушная академия им. Н. Е. Жуковского и Ю. А. Гагарина», 2012.

Шепеть Игорь Петрович

Технологический институт сервиса -

филиал Донского государственного технического университета, Ставрополь E-mail: ship.1963@mail.ru

Литвин Дмитрий Борисович

Ставропольский государственный аграрный университет

E-mail: litvin-372@yandex.ru 